\section{(- OPEN ACCESS}

\title{
Arterial stiffness association with chronic inflammatory disorders in the UK Biobank study
}

\author{
Alex Dregan ${ }^{1,2}$
}

- Additional material is published online only. To view please visit the journal online (http://dx.doi.org/10.1136/ heartjnl-2017-312610).

${ }^{1}$ School of Population Health Sciences, King's College London, London, UK

${ }^{2} \mathrm{NIHR}$, Biomedical Research Centre at Guy's and St Thomas NHS Foundation Trust, London, UK

\section{Correspondence to}

Dr Alex Dregan, King's College London, Department of

Primary Care and Public Health Sciences, 3rd Floor Addison House, London, SE1 1UL, UK ; alexandru.dregan@kcl.ac.uk

Received 23 October 2017 Revised 6 December 2017 Accepted 6 December 2017 Published Online First 4 January 2018
Check for updates

To cite: Dregan A. Heart 2018:104:1257-1262.

\begin{abstract}
Objective The present study tested the hypothesis that arterial stiffness will be elevated across overall and specific inflammatory disorders compared with an inflammation-free comparison group.
\end{abstract}

Methods Adults $(n=171125)$ aged $40-70$ years from the UK Biobank who were cardiovascular disease (CVD) free and who had their arterial stiffness assessed at the time of study recruitment between 2006 and 2010 were included. The main exposure was represented by a global measure of chronic inflammatory disorders. Two inflammatory biomarker measures (eg, leucocytes count, granulocytes count) were included as markers of inflammation severity. The arterial stiffness index assessed by a non-invasive technique represented the study primary outcome measure.

Results A total of 5976 (3\%) participants diagnosed with inflammatory disorders and 165149 participants without an inflammatory disorder had data on arterial stiffness. Adjusted linear regression analyses revealed a 14\% increment in mean arterial stiffness for chronic inflammatory disorders (beta coefficient ( $\beta$ ) 1.14, 95\% Cl 1.05 to $1.24, P=0.002$ ) compared with no chronic inflammatory disorder. Arterial stiffness tended to increase ( $P$ value $=0.031$ ) with tertiles of leucocytes and granulocytes count. For instance, mean arterial stiffness values increased from $1.11(95 \% \mathrm{Cl} 0.96$ to 1.29$)$ in the first tertile to $1.17(95 \% \mathrm{Cl} 1.02$ to 1.34$)$ in the second tertile, and $1.21(95 \% \mathrm{Cl} 1.05$ to 1.39$)$ in the third tertile of leucocytes count. There was evidence for similar associations with some of the most common individual inflammatory disorders, including psoriasis and rheumatoid arthritis.

Conclusion Arterial stiffness was associated with multiple chronic inflammatory disorders. An increasing trend in mean arterial stiffness was also documented with increasing tertiles of different inflammatory biomarkers. Future studies are needed to investigate the discriminant value of arterial stiffness to predict major CVD events within various inflammatory disorders.

Cardiovascular diseases (CVD) represent a major burden among people diagnosed with chronic inflammatory disorders. For instance, several inflammatory disorders (eg, rheumatoid arthritis (RA), psoriasis and inflammatory bowel disorders (IBD)) were found to confer a $50 \%$ increased risk of major vascular events (eg, myocardial infarction (MI), stroke) compared with the general population. ${ }^{1-3}$ In addition to traditional vascular risk factors (eg, hypertension, dyslipidaemia, obesity, smoking), inflammation has been documented as a major determinant of CVD risk across diverse inflammatory disorders. ${ }^{1}$ These findings emphasise the importance of identifying the pathways through which chronic inflammation may lead to early CVD pathogenesis. Studies with small (mainly hospital-based) samples point towards a potential association between inflammation with arterial stiffness, an independent predictor of future CVD events. ${ }^{4-6}$ Research in this area remains primarily exploratory, however, with studies employing diverse non-invasive measures of arterial stiffness (eg, pulse wave velocity (PWV), augmentation index, stiffness index (SI)) across different populations, making it difficult to draw robust overall conclusions. $^{7-9}$ The UK Biobank study includes several physiological and imaging techniques that may facilitate early identification of future risk of CVDs. For instance, the study includes an SI measure as a surrogate marker for subclinical atherosclerosis. While studies with small healthy populations indicate moderate correlation between the SI and the $\mathrm{PWV},{ }^{10}$ no studies have evaluated the prognostic value of SI within diverse inflammatory disorders. Recently, the study added data on leucocytes count that have been used as markers of inflammation severity. It has been documented, for instance, that leucocytes release cytokines, triggering further macrophage recruitment and the proliferation of smooth muscle cells within the vascular wall. ${ }^{11}$ In addition, protease secretion leads to endothelial damage of the coronary vessels, exposing thrombogenic collagen and predisposing the vessels to thrombus formation. Phagocytes release myeloperoxidase, which generates reactive oxygen species that are involved in the generation and progression of atherosclerosis and that contribute to the development of plaque instability in acute MI. ${ }^{11}$ Whether chronic inflammation is associated with a surrogate marker of arterial stiffness is not clearly established. The present study aimed to address this concern within a large community-based sample of adults aged 40-69 years. The study's main hypothesis was that arterial stiffness would be more common among inflammatory disorders relative to people in the general population, and that this association would vary with disease severity.

\section{METHODS \\ Data}

The data for the present study come from the UK Biobank, a large population-based prospective study developed to facilitate detailed investigations about the determinants of diseases at population level. The UK Biobank collects detailed data from 
over 500000 participants aged 40-69, including lifestyle, demographics, clinical diagnoses, treatment, lab tests (ie, biomarkers), imaging and genotype information. ${ }^{12}$ A more detailed description of the UK Biobank data is provided elsewhere. ${ }^{12}$

The present study was restricted to a subset of study participants $(n=171125)$ with measured arterial stiffness at baseline. Participants diagnosed with RA, systemic lupus erythematosus (SLE), Sjogren syndrome, psoriasis, ankylosing spondylitis (AS), systemic vasculitis or IBD represented the exposed group. Participants free of these diagnoses represent the comparison group. Following Shen et $a l^{13}$ the study excluded participants with a history of CVDs (including coronary heart disease, stroke and peripheral arterial disease) or type II diabetes. Because arterial stiffness represents a blood pressure independent predictor of CVD events, ${ }^{14}$ patients with previous hypertension have been included in the analyses. This procedure allowed to account for the potential modulatory role of antihypertensive therapies on arterial stiffness. ${ }^{15}$ All participants provided written informed consent.

\section{Arterial stiffness outcomes}

A photoplethysmograph transducer was placed on the index finger of the participant's dominant hand and used to calculate an arterial SI. The SI was assessed as the height of the participants divided by the time between the first (systolic) and second (diastolic) wave peaks, and was expressed in metres per second $(\mathrm{m} / \mathrm{s})$. SI is a clinical marker of larger artery stiffness and has been found to be moderately correlated with PWV-the gold standard measure of arterial stiffness. ${ }^{10}$ A higher SI is considered indicative of stiffer arteries.

\section{Exposures}

The study's main exposure was a global measure of chronic inflammatory disorders, including RA, psoriasis, IBD, SLE, Sjogren syndrome, systemic vasculitis and AS. These measures were developed from participants' self-reports and were recorded according to the International Classification of Diseases, 10th Revision criteria. To explore potential variation within specific inflammatory disorders, separate binary variables (yes/no) were developed for the most common inflammatory disorders, specifically RA, IBD and psoriasis. Leucocytes count was used to categorise participants into tertiles of inflammation disease severity. A composite granulocytes count-based inflammation severity measure was also developed. This measure classified participants into tertiles based on the average count of neutrophil, basophil and eosinophil biomarkers. An inflammation duration variable, which classified participants into tertiles of disorder duration, was used for sensitivity analyses.

\section{Covariates}

The study covariates included sociodemographic characteristics, specifically age (continuous measure), gender (female vs male), ethnicity (White, Black, Asian, Other) and deprivation. Deprivation was based on Townsend deprivation indices derived from aggregated data on car ownership, household overcrowding, owner occupation and unemployment (higher scores represent higher degree of deprivation). The study also adjusted for traditional vascular risk factors, including smoking (never, ex, current smoker), hypertension (yes/no), hypercholesterolaemia (yes/no) and body mass index (BMI; $\mathrm{kg} / \mathrm{m}^{2}$ ). Antihypertensive and lipid-lowering drugs (yes/no) were also included as covariates. Non-steroidal anti-inflammatory drugs (NSAID) and corticosteroid drugs were included as separate binary covariates (they may have different impact on leucocytes count) due to their association with increased CVD risk. ${ }^{16}$ Sensitivity analyses also adjusted for disease-modifying antirheumatic drug (DMARD) prescribing.

\section{Statistical analysis}

Descriptive analyses (eg, frequencies, means) were used to describe baseline characteristics among participants diagnosed with inflammatory disorders and the comparison group. The associations between inflammatory disorders with the SI were estimated using multivariable linear regression analyses with robust SE. Separate estimation models were performed for overall and specific inflammatory disorder (eg, RA, psoriasis and IBD). All models adjusted for age, sex, deprivation, BMI, smoking, ethnicity, previous diagnoses of hypertension and hypercholesterolaemia, anti-inflammatory drugs, as well as antihypertensive and lipid-lowering medications. Analyses considering multiple imputation provided similar estimates to the main estimation models and only the latter are presented here. To test for potential dose-response associations, linear regression was used to compare inflammatory participants in different leucocytes count tertiles to the reference group of no chronic inflammatory disorder. ${ }^{1}$ Sensitivity analyses stratified by inflammation disorder were also performed. Additional sensitivity analyses exploring the association between inflammatory disorder duration with the SI measure and adjusting for DMARDs were performed. The study did not adjust for multiple comparisons as it could increase the risk of type II errors. ${ }^{17}$ Results are reported as $\beta$ estimates with 95\% CIs. All analyses were conducted using STATA V.14 software (regress command with the robust option), using a $P$ value of 0.05 as the threshold for statistical significance.

\section{RESULTS}

After excluding participants with a diagnosis of CVD or diabetes prior to SI assessment, the analyses included 5976 participants diagnosed with an inflammatory disorder and 165149 participants without an inflammatory disorder. The most common disorder was psoriasis $(n=2019)$, followed by RA $(n=1679)$ and $\operatorname{IBD}(n=1392)$. For all groups, the distribution of age was similar (table 1). Women were over-represented across all conditions, except psoriasis (48\%). Participants diagnosed with chronic inflammatory disorders presented with higher prescribing rates of NSAIDs (25\%), corticosteroids (14\%), DMARDs (18\%) and antihypertensive $(21 \%)$ drugs relative to the comparison group (18\%, 3\%, 0\% and 16\%, respectively).

Figure 1 (see online supplementary figure S1 for data on all inflammatory disorders) illustrates the adjusted and unadjusted mean SI values for chronic inflammatory participants and their comparison group. Mean SI values were higher in the group $(9.46,95 \%$ CI 9.38 to 9.53$)$ relative to the comparison group (9.32, 95\% CI 9.31 to 9.34). Also, RA (9.47, 95\% CI 9.33 to 9.62), IBD $(9.48,95 \%$ CI 9.33 to 9.63$)$ and psoriasis $(9.49$, $95 \%$ CI 9.36 to 9.61 ) disorders presented with the highest mean values for the SI.

Multivariable linear regression analyses (table 2) revealed that chronic inflammatory disorders were associated with $14 \%$ increase in mean arterial stiffness value $(\beta=1.14,95 \% \mathrm{CI}$ 1.05 to $1.25, \mathrm{P}=0.002$ ) compared with no chronic inflammatory disorder. Mean SI values increased gradually with tertiles of the composite biomarkers of inflammation. For instance, the SI increased from $11 \%$ in the first tertile $(95 \%$ CI 0.96 to $1.29, \mathrm{P}=0.162)$ to $17 \%$ in the second tertile $(95 \% \mathrm{CI} 1.02$ to $1.34, \mathrm{P}=0.029)$, and $21 \%$ in the third tertile $(1.21,95 \% \mathrm{CI}$ 
Table 1 Participants' characteristics at baseline assessment

\begin{tabular}{|c|c|c|c|c|c|}
\hline & All (5976) & $\mathrm{RA}(\mathrm{n}=1672)$ & Psoriasis (2091) & IBD (1392) & Unexposed (165 149) \\
\hline Age, mean (SD) & $58(8)$ & $59(7)$ & $57(8)$ & $57(8)$ & $57(8)$ \\
\hline Gender, female & $3486(58)$ & $1173(70)$ & $1013(48)$ & $783(56)$ & $93312(55)$ \\
\hline Deprivation, mean (SD) & $-1.17(3)$ & $-1.06(3)$ & $-1.10(3)$ & $-1.31(3)$ & $-1.21(3)$ \\
\hline \multicolumn{6}{|l|}{ Ethnicity } \\
\hline White & $5726(94)$ & $1534(92)$ & $1988(95)$ & $1320(95)$ & 150412 (92) \\
\hline Asian & $183(3)$ & $68(4)$ & $56(3)$ & $37(3)$ & $5368(3)$ \\
\hline Black & $76(1)$ & $31(2)$ & $8(1)$ & $11(1)$ & $4913(3)$ \\
\hline Other & $100(2)$ & $34(2)$ & $31(1)$ & $19(1)$ & $4024(2)$ \\
\hline \multicolumn{6}{|l|}{ Smoker } \\
\hline Never & $2921(49)$ & $827(50)$ & $979(47)$ & $683(49)$ & $93508(57)$ \\
\hline Ex & $2344(39)$ & $654(39)$ & $805(39)$ & $586(42)$ & $54919(33)$ \\
\hline Current & $686(12)$ & $183(11)$ & $298(14)$ & $120(9)$ & $15813(10)$ \\
\hline BMI, mean (SD), $\mathrm{kg} / \mathrm{m}^{2}$ & $27.65(5)$ & $27.72(5)$ & $28.11(5)$ & $26.98(4)$ & $27.24(5)$ \\
\hline Antihypertensive drugs & $1292(21)$ & $390(23)$ & $414(20)$ & $257(18)$ & $27127(16)$ \\
\hline Statins & $748(12)$ & $238(14)$ & $254(12)$ & $142(10)$ & $17619(11)$ \\
\hline NSAIDs & $1535(25)$ & $600(36)$ & $451(22)$ & $211(15)$ & $29636(18)$ \\
\hline Corticosteroids & 866 (14) & $266(13)$ & $166(12)$ & $108(8)$ & $4297(3)$ \\
\hline DMARDs & $1125(18)$ & $694(42)$ & $168(8)$ & $162(12)$ & $301(0)$ \\
\hline Hypertension & $1830(30)$ & $542(32)$ & $611(29)$ & $349(25)$ & $41984(25)$ \\
\hline Hypercholesterolemia & 809 (13) & $229(14)$ & $305(15)$ & $136(10)$ & $20726(13)$ \\
\hline
\end{tabular}

Figures are numbers and percentages unless otherwise specified.

DMARD, disease-modifying antirheumatic drug; IBD, inflammatory bowel disorder; NSAID, non-steroidal anti-inflammatory drug; RA, rheumatoid arthritis.

1.05 to $1.39, \mathrm{P}=0.009$ ) of leucocytes count. Postestimation analyses revealed that the differences in mean arterial stiffness between the three leucocyte tertiles were statistically significant $(\mathrm{P}$ value $=0.031)$. A similar pattern emerged with regard to the composite granulocytes count measure; however, the association was statistically significant only at the highest tertile of granulocytes count $(1.23,95 \%$ CI 1.06 to $1.42, \mathrm{P}=0.005)$.

Mean SI was also increased within RA $(1.18$, 95\% CI 1.01 to $1.39, \mathrm{P}=0.044)$ and psoriasis $(1.21,95 \% \mathrm{CI} 1.03$ to 1.32 , $\mathrm{P}=0.016)$, but not IBD $(1.14,95 \%$ CI 0.98 to $1.35, \mathrm{P}=0.091)$ disorders (table 3). A statistically significant association between severity of inflammation with mean SI was revealed within both RA and psoriasis disorders. In adjusted analyses, however, the association was statistically significant only at the highest tertile of granulocytes count for psoriasis $(1.29,95 \%$ CI 1.03 to 1.60 , $\mathrm{P}<0.001)$.

\section{Sensitivity analyses}

Analyses that stratified participants by inflammation disorder revealed a gradual increment in mean SI with higher tertiles of leucocytes count. The increase was statistically significant in the reference group but not in the chronic inflammation group, possibly due to insufficient power for the latter. SI values increased linearly from the first to the third tertile of inflammatory disorder duration (years since diagnosis), being statistically

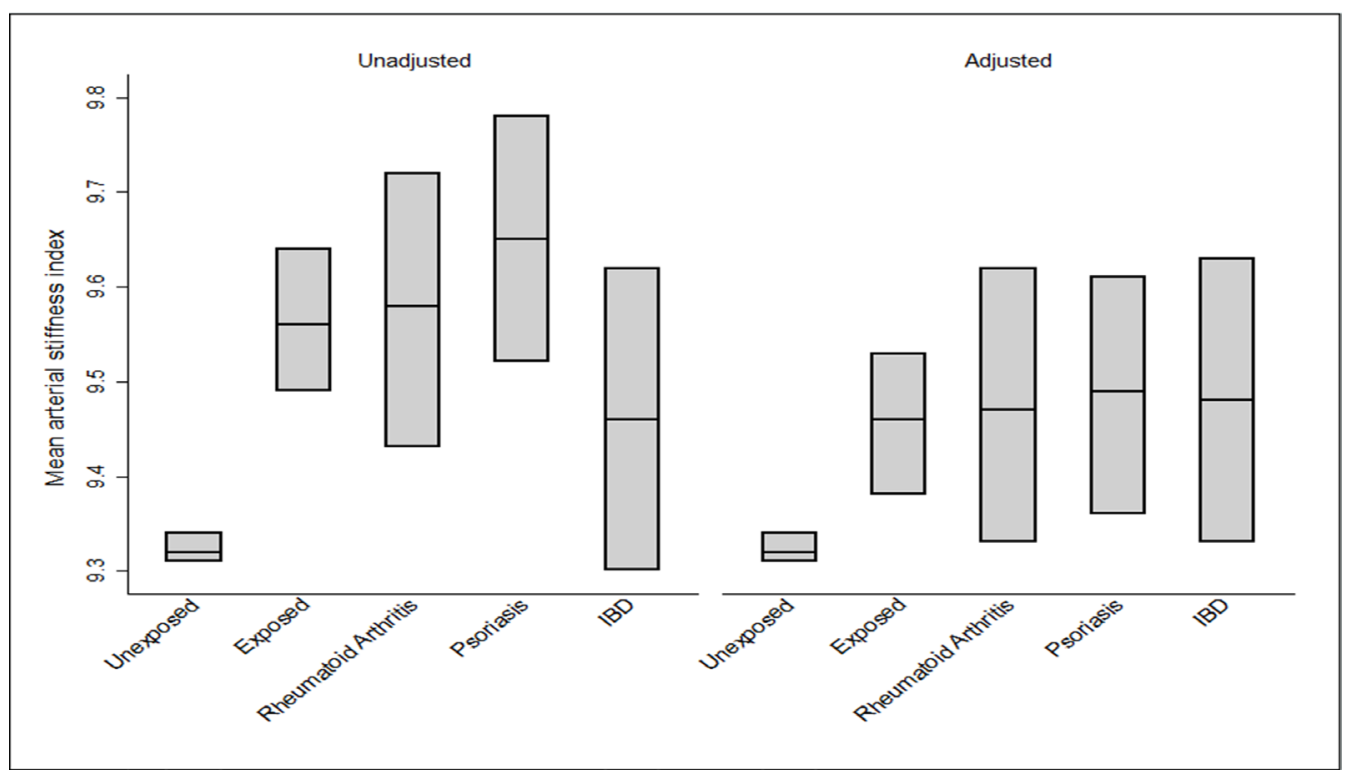

Figure 1 Unadjusted and adjusted mean values for the arterial stiffness index across the study groups. IBD, inflammatory bowel disorder. 
Table 2 Exponentiated coefficients and associated $95 \% \mathrm{Cl}$ for the association between overall chronic inflammatory disorders and inflammation severity with the arterial stiffness index

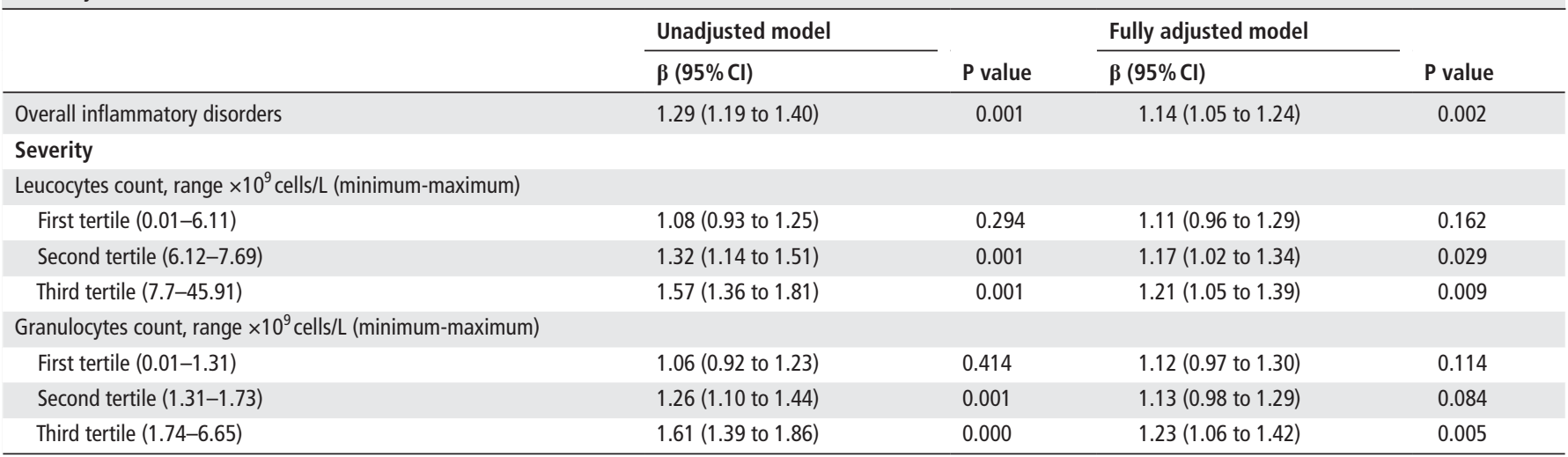

$\beta$, exponentiated coefficient.

significant at the middle $(1.16,95 \%$ CI 1.01 to $1.34, \mathrm{P}=0.038)$ and highest tertiles $(1.18,95 \% \mathrm{CI} 1.03$ to $1.35, \mathrm{P}=0.019)$ of disorder duration (online supplementary table S1). A similar pattern was also apparent between the SI with RA and IBD disorder duration. Finally, adjusting for DMARD prescribing did not alter the direction or statistical significance of associations.

\section{DISCUSSION}

Using a large community sample, an overall measure of major chronic inflammatory disorders was associated with higher rates of arterial stiffness. The association appeared to increase with the severity of chronic inflammation, as assessed by a composite measure of inflammatory biomarkers (eg, leucocytes count and granulocytes count). Within each composite measure, for instance, the association with arterial stiffness increased gradually from the first to the third tertile. These findings support the possibility of a dose-response association between inflammation severity with a non-invasive measure of arterial stiffness.

Inflammatory disorder-specific analyses supported a modest association between arterial stiffness with psoriasis and RA disorders, but less so with regard to IBDs. This suggestion was supported by the evidence that the SI increased with each tertile of granulocytes count within the psoriasis disorder. No clear dose-response relationship was observed with regard to the leucocytes count measure, implying that leucocytes count may be an insensitive and non-specific marker of inflammation severity or that the study was insufficiently powered for disorder-specific analyses. The UK Biobank's intention to incorporate biomarker data on C-reactive protein (CRP) and rheumatoid factor would offer the opportunity to compare the prognostic value of leucocytes count with these established markers of chronic inflammation.

While specific chronic inflammatory disorders may vary with regard to clinical presentation and underlying risk factors, ${ }^{18}$ they all have in common elevated levels of low-grade inflammation. These heterogeneous conditions also present similar rates of cardiovascular complications, including major CVD events and atherosclerosis. These associations may vary with inflammation severity, and the present study findings documented the potential value of using composite measures of markers of chronic inflammation for these purposes. The extent to which these findings are translated to specific chronic inflammatory disorders remains to be determined, however.

A growing number of studies suggest that inflammation could be responsible for part of the excess cardiovascular risk observed in patients with chronic inflammatory diseases. Recently, it was reported that, compared with control subjects, carotid-femoral PWV and augmentation index were significantly higher in patients with chronic inflammation, including inflammatory bowel diseases (IBD), RA, SLE and systemic sclerosis. ${ }^{19-21}$ Moreover, a significant relationship between aortic stiffness and left ventricular systolic and diastolic dysfunction was reported in

Table 3 Exponentiated coefficients and associated $95 \%$ Cls for the association between specific chronic inflammatory disorders and inflammation severity with the arterial stiffness index

\begin{tabular}{|c|c|c|c|c|c|c|}
\hline & \multicolumn{2}{|l|}{ Rheumatoid arthritis } & \multicolumn{2}{|l|}{ Psoriasis } & \multicolumn{2}{|c|}{ Inflammatory bowel disorders } \\
\hline & $\begin{array}{l}\text { Unadjusted } \\
\boldsymbol{\beta}(95 \% \mathrm{Cl}) \\
\end{array}$ & $\begin{array}{l}\text { Adjusted } \\
\boldsymbol{\beta}(95 \% \mathrm{Cl})\end{array}$ & $\begin{array}{l}\text { Unadjusted } \\
\boldsymbol{\beta}(95 \% \mathrm{Cl}) \\
\end{array}$ & $\begin{array}{l}\text { Adjusted } \\
\boldsymbol{\beta}(95 \% \mathrm{Cl})\end{array}$ & $\begin{array}{l}\text { Unadjusted } \\
\boldsymbol{\beta}(95 \% \mathrm{Cl})\end{array}$ & $\begin{array}{l}\text { Adjusted } \\
\boldsymbol{\beta}(95 \% \mathrm{Cl})\end{array}$ \\
\hline Disorder & 1.29 (1.09 to 1.52$)$ & 1.18 (1.01 to 1.39$)$ & 1.39 (1.22 to 1.58$)$ & 1.17 (1.03 to 1.32$)$ & 1.15 (0.98 to 1.36$)$ & $1.15(0.98$ to 1.35$)$ \\
\hline \multicolumn{7}{|l|}{ Severity } \\
\hline \multicolumn{7}{|c|}{ Leucocytes count tertiles } \\
\hline First & 1.19 (0.88 to 1.60$)$ & 1.20 (0.90 to 1.61$)$ & $1.03(0.82$ to 1.29$)$ & 1.02 (0.82 to 1.28$)$ & $1.08(0.81$ to 1.45$)$ & $1.17(0.88$ to 1.55$)$ \\
\hline Second & 1.24 (0.93 to 1.64$)$ & 1.16 (0.88 to 1.52$)$ & 1.66 (1.33 to 2.08$)$ & 1.36 (1.09 to 1.69$)$ & 1.24 (0.93 to 1.66$)$ & $1.24(0.94$ to 1.65$)$ \\
\hline Third & 1.59 (1.19 to 2.13$)$ & 1.25 (0.93 to 1.67$)$ & 1.65 (1.32 to 2.07$)$ & 1.21 (0.97 to 1.50$)$ & 1.18 (0.89 to 1.56$)$ & $1.10(0.83$ to 1.45$)$ \\
\hline \multicolumn{7}{|c|}{ Granulocytes count tertiles } \\
\hline First & 1.14 (0.85 to 1.52$)$ & 1.23 (0.91 to 1.67$)$ & 1.12 (0.89 to 1.42 ) & 1.13 (0.90 to 1.42 ) & 1.14 (0.85 to 1.53$)$ & $1.26(0.94$ to 1.69$)$ \\
\hline Second & $1.33(1.00$ to 1.75$)$ & 1.24 (0.93 to 1.65$)$ & 1.45 (1.16 to 1.81$)$ & 1.18 (0.95 to 1.47$)$ & 1.19 (0.90 to 1.58$)$ & $1.21(0.92$ to 1.59$)$ \\
\hline Third & 1.56 (1.16 to 2.11$)$ & $1.31(0.96$ to 1.80$)$ & 1.71 (1.37 to 2.15$)$ & $1.29(1.03$ to 1.60$)$ & 1.19 (0.89 to 1.59$)$ & $1.09(0.82$ to 1.45$)$ \\
\hline
\end{tabular}

$\beta$, exponentiated coefficient. 
patients with IBD. ${ }^{22}$ This finding may explain the link between increased aortic stiffness with CVD events in patients with IBD and why these patients present with an increased CVD risk despite a low prevalence of classical cardiovascular risk factors.

Empirical evidence about differences in arterial stiffness between diverse chronic inflammatory disorders and the general population is unavailable, precluding any direct comparison between this study findings with previous research. Nevertheless, the findings for increased arterial stiffness associated with psoriasis and RA disorders are in line with earlier evidence with smaller samples and different surrogate markers of arterial stiffness. ${ }^{152324}$ Booth et $a l^{25}$ found increased arterial stiffness among 31 patients diagnosed with systemic vasculitis, but the association was limited to patients $(n=15)$ with active disease. The current study findings for a statistically significant association between arterial stiffness with the highest tertiles of leucocytes and granulocytes count point towards similar evidence across multiple chronic inflammatory disorders. The present study findings appear to support earlier evidence for a lack of association between arterial stiffness with IBDs, ${ }^{26}$ but this suggestion needs to be interpreted within the constraints of insufficient statistical power. Recent meta-analyses ${ }^{20} 21$ reported increased augmentation index and carotid-femoral PWV among patients diagnosed with IBD. The higher, but not statistically significant, SI for IBD condition in this study may be explained by the use of a surrogate marker of arterial stiffness or the remission of disease in participants diagnosed with IBD. These explanations may also account for the lack of association between white blood cells with the SI in this study, which is not in line with suggestions from a recent meta-analysis. ${ }^{22}$

\section{Strengths and limitations}

The present study has several strengths, including large population sample, comprehensive phenotype data, physiological measures of inflammatory biomarkers and objectively assessed arterial stiffness. As with most observational studies, several limitations need consideration in interpreting the study findings. The UK Biobank data are based on self-reported chronic inflammatory disorders, which may increase the risk of misclassification bias. If inflammatory disorder diagnosis was over-reported or under-reported, this bias may slightly attenuate or strengthen the association with arterial stiffness. The prevalence of chronic inflammatory disorders in this study was similar to earlier investigations, ${ }^{1}$ suggesting minimal misclassification bias. The estimation models were limited by the UK Biobank data available at time of study, and direct measures of disease activity (eg, disease activity score) or direct measures of arterial stiffness could not be considered. The present study analyses used composite measures of inflammatory biomarkers (eg, leucocytes, granulocytes), as surrogate markers of inflammation severity. These biomarkers have been documented in the past to differentiate between the preclinical and clinical phases on CVDs. ${ }^{27}$ Plans to include CRP and rheumatoid factor measures into the UK Biobank data would provide for future validation studies with more direct biomarker measurements. As with any observational data, the health survivor bias is a possibility within the UK Biobank. This limitation is of a greater concern for generalising the findings about disease prevalence, and less so about generalising the effect size estimates. ${ }^{28}$ The SI, as assessed by the PulseTrace technique, was found to be only moderately correlated with PWV. ${ }^{29}$ The statistically significant associations and the documented dose-response relationship appear to support a potential role of the SI in the context of inflammatory disorders. This suggestion needs confirmation with future prospective studies, however. Notably, the moderate correlation between SI with PWV observed in Salvi et al's study ${ }^{29}$ could be due to the study insufficient statistical power $(\mathrm{n}=50)$ or limited generalisability (just one participant presented a diagnosis of an inflammatory disorder). Information on left ventricular dysfunction, known to be associated with inflammation and CVD risk, ${ }^{30}$ was not available in the data. The linkage of the UK Biobank data with patients' medical records in the near future would allow for more detailed investigations, including the association between increased arterial stiffness and CVD events in patients diagnosed with chronic inflammation. It would also provide for other investigations, including the impact of anti-tumour necrosis factor therapy on the arterial SI and for validating the diagnosis of chronic inflammatory disorders. The study data were underpowered to provide robust estimates on the association between arterial stiffness with other specific CIs (eg, SLE, AS, systemic vasculitis). Preliminary analyses appeared supportive of increased arterial stiffness within AS and vasculitis disorders, but these findings need validation with larger samples.

\section{CONCLUSIONS}

Overall, this study findings support a potential association between markers of chronic inflammation with an estimate of arterial stiffness. The study findings also document a potential role of composite measures of inflammatory biomarkers to discriminate among people diagnosed with inflammatory disorders at different risk of future CVDs. Whether the addition of composite measures of inflammatory biomarkers to traditional CVD risk scores (eg, QRISK2, Framingham) improves the latter discriminant and predictive values within chronic inflammatory disorders awaits further confirmation from prospective studies. Plans to include an MRI-based measure of PWV on the full UK Biobank sample would offer the opportunity to further validate the accuracy of the SI as an estimate of arterial stiffness within the context of chronic inflammatory disorders.

\section{Key messages}

What is already known on this subject? Patients diagnosed with chronic inflammatory disorders are at increased risk of major cardiovascular events. Strategies to facilitate early identification of subclinical atherosclerosis in chronic inflammation have been proposed over the past years.

What might this study add?

Arterial stiffness index was increased among participants diagnosed with chronic inflammatory, including rheumatoid arthritis and psoriasis. The association varied with the distribution of inflammatory biomarkers as markers of inflammation severity.

How might this impact on clinical practice? The stiffness index, a non-invasive measure of arterial stiffness, may provide additional prognostic and discriminatory value for future cardiovascular disease risk prediction within overall and specific chronic inflammatory disorders. 
Acknowledgements The author would like to acknowledge Professor Martin Gulliford valuable comments and suggestions during the preparation of the manuscript.

Contributors The author is a single contributor to developing the study research question, data access, data analysis, and drafting the manuscript and write-up of the final version.

Funding This study has been conducted using the UK Biobank resource. UK Biobank was established by the Wellcome Trust medical charity, Medical Research Council, Department of Health, Scottish Government and the Northwestern Regional Development Agency. It has also had funding from the Welsh Assembly and the British Heart Foundation. AD is supported by the National Institute for Health Research Biomedical Research Center at Guy's and St Thomas' National Health Service Foundation Trust and King's College London.

Disclaimer The views expressed are those of the author and not necessarily those of the National Health Service, the National Institute of Health Research, or the Department of Health.

Competing interests None declared.

Ethics approval Ethical approval was obtained by the data holders from the National Health Service National Research Ethics Service (Ref: 11.NW/0382).

Provenance and peer review Not commissioned; externally peer reviewed.

Open access This is an open access article distributed in accordance with the terms of the Creative Commons Attribution (CC BY 4.0) license, which permits others to distribute, remix, adapt and build upon this work, for commercial use, provided the original work is properly cited. See: http://creativecommons.org/licenses/by/4.0/

(c) Article author(s) (or their employer(s) unless otherwise stated in the text of the article) 2018. All rights reserved. No commercial use is permitted unless otherwise expressly granted.

\section{REFERENCES}

1 Dregan A, Charlton J, Chowienczyk P, et al. Chronic inflammatory disorders and risk of type 2 diabetes mellitus, coronary heart disease, and stroke: a population-based cohort study. Circulation 2014;130:837-44.

2 Ahlehoff 0 , Gislason GH, Charlot M, et al. Psoriasis is associated with clinically significant cardiovascular risk: a Danish nationwide cohort study. J Intern Med 2011:270:147-57.

3 Dregan A, Chowienczyk P, Molokhia M. Cardiovascular and type 2 diabetes morbidity and all-cause mortality among diverse chronic inflammatory disorders. Heart 2017; 103:1867-73.

4 Sacre K, Escoubet B, Pasquet B, et al. Increased arterial stiffness in systemic lupus erythematosus (SLE) patients at low risk for cardiovascular disease: a cross-sectional controlled study. PLoS One 2014:9:e94511.

5 Zanoli L, Cannavò M, Rastelli S, et al. Arterial stiffness is increased in patients with inflammatory bowel disease. J Hypertens 2012;30:1775-81.

6 Humphreys JH, Warner A, Chipping J, et al. Mortality trends in patients with early rheumatoid arthritis over 20 years: results from the Norfolk Arthritis Register. Arthritis Care Res 2014:66:1296-301.

7 Mäki-Petäjä KM, Hall FC, Booth AD, et al. Rheumatoid arthritis is associated with increased aortic pulse-wave velocity, which is reduced by anti-tumor necrosis factoralpha therapy. Circulation 2006;114:1185-92.

8 Dzieża-Grudnik A, Sulicka J, Strach M, et al. Arterial stiffness is not increased in patients with short duration rheumatoid arthritis and ankylosing spondylitis. Blood Press 2017:26:115-21.
9 Ambrosino P, Tasso M, Lupoli R, et al. Non-invasive assessment of arterial stiffness in patients with rheumatoid arthritis: a systematic review and meta-analysis of literature studies. Ann Med 2015;47:457-67.

10 Woodman RJ, Kingwell BA, Beilin LJ, et al. Assessment of central and peripheral arterial stiffness: studies indicating the need to use a combination of techniques. $A m$ Hypertens 2005:18:249-60.

11 Madjid M, Fatemi O. Components of the complete blood count as risk predictors for coronary heart disease: in-depth review and update. Tex Heart Inst J 2013:40:17-29.

12 Sudlow C, Gallacher J, Allen N, et al. UK biobank: an open access resource for identifying the causes of a wide range of complex diseases of middle and old age. PLoS Med 2015;12:e1001779.

13 Shen J, Shang Q, Li EK, et al. Cumulative inflammatory burden is independently associated with increased arterial stiffness in patients with psoriatic arthritis: a prospective study. Arthritis Res Ther 2015;17:75.

14 Dudenbostel T, Glasser SP. Effects of antihypertensive drugs on arterial stiffness. Cardiol Rev 2012;20:259-63.

15 Gisondi P, Fantin F, Del Giglio M, et al. Chronic plaque psoriasis is associated with increased arterial stiffness. Dermatology 2009;218:110-3.

16 Arfè A, Scotti L, Varas-Lorenzo C, et al. Non-steroidal anti-inflammatory drugs and risk of heart failure in four European countries: nested case-control study. BMJ 2016;354:i4857.

17 Feise RJ. Do multiple outcome measures require p-value adjustment? BMC Med Res Methodol 2002;2:8

18 Wijmenga C. Expressing the differences between Crohn disease and ulcerative colitis. PLoS Med 2005;2:e230

19 Wu GC, Liu HR, Leng RX, et al. Subclinical atherosclerosis in patients with systemic lupus erythematosus: a systemic review and meta-analysis. Autoimmun Rev 2016;15:22-37.

20 Wu GC, Leng RX, Lu Q, et al. Subclinical atherosclerosis in patients with inflammatory bowel diseases: a systematic review and meta-analysis. Angiology 2017:68:447-61.

21 Zanoli L, Granata A, Lentini P, et al. Augmentation index is increased in patients with inflammatory bowel disease, a meta-analysis. Eur J Intern Med 2017:39:e31-e32.

22 Zanoli L, Boutouyrie P, Fatuzzo P, et al. Inflammation and aortic stiffness: an individual participant data meta-analysis in patients with inflammatory bowel disease. J Am Heart Assoc 2017:6:e007003.

23 Botta E, Meroño T, Saucedo C, et al. Associations between disease activity, markers of HDL functionality and arterial stiffness in patients with rheumatoid arthritis. Atherosclerosis 2016;251:438-44.

24 Roman MJ, Devereux RB, Schwartz JE, et al. Arterial stiffness in chronic inflammatory diseases. Hypertension 2005;46:194-9.

25 Booth AD, Wallace S, McEniery CM, et al. Inflammation and arterial stiffness in systemic vasculitis: a model of vascular inflammation. Arthritis Rheum 2004; 50:581-8.

26 Fan F, Galvin A, Fang L, et al. Comparison of inflammation, arterial stiffness and traditional cardiovascular risk factors between rheumatoid arthritis and inflammatory bowel disease. J Inflamm 2014:11:29.

27 Grossmann V, Schmitt VH, Zeller T, et al. Profile of the immune and inflammatory response in individuals with prediabetes and type 2 diabetes. Diabetes Care 2015;38:1356-64

28 Fry A, Littlejohns TJ, Sudlow C, et al. Comparison of sociodemographic and health-related characteristics of uk biobank participants with those of the general population. Am J Epidemiol 2017;186:1026-34.

29 Salvi P, Magnani E, Valbusa F, et al. Comparative study of methodologies for pulse wave velocity estimation. J Hum Hypertens 2008;22:669-77.

30 Masiha S, Sundström J, Lind L. Inflammatory markers are associated with left ventricular hypertrophy and diastolic dysfunction in a population-based sample of elderly men and women. J Hum Hypertens 2013;27:13-17. 